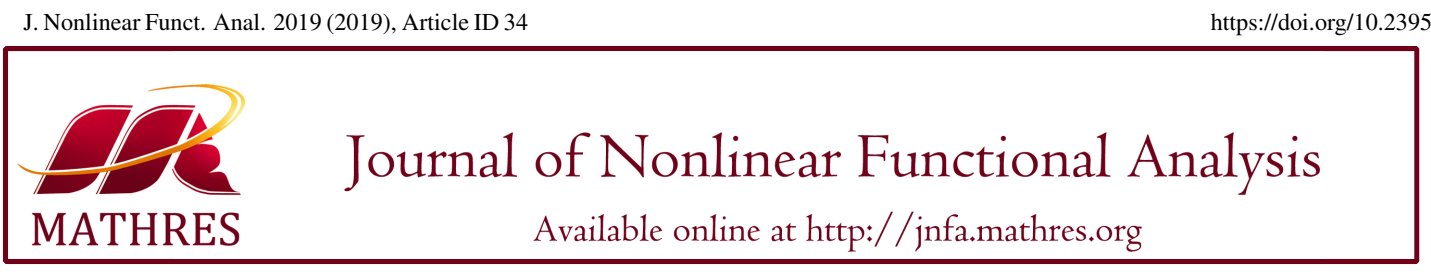

https://doi.org/10.23952/jnfa.2019.34

\title{
HOPF BIFURCATION FORMULAE AND APPLICATIONS TO THE GENESIO-TESI SYSTEM
}

\author{
BO SANG \\ School of Mathematical Sciences, Liaocheng University, Liaocheng 252059, China
}

\begin{abstract}
The purpose of this paper is to propose some formulae for Hopf bifurcation analysis, and investigate applications to a chaotic system. We perform a substantial simplification for the classical Hopf bifurcation formulae. Our results can be extended to multi-dimensional quadratic systems. As an application, we consider the Genesio-Tesi system. Finally, the dynamics of the system are analyzed by bifurcation diagrams, Lyapunov exponents, phase portraits and Poincaré maps. We show that the system can generate chaos via a Hopf bifurcation and period doubling cascade as the control parameter varies. Some other bifurcations can be observed, which includes saddle-node bifurcations, interior crises and boundary crises.
\end{abstract}

Keywords. Chaos; Genesio-Tesi system; Hopf bifurcation; Limit cycle.

2010 Mathematics Subject Classification. 34C05, 34C07, 37G15.

\section{INTRODUCTION}

Periodic oscillations are observed in many naturally occurring non-conservative systems. These phenomena are often related to the occurrence of Hopf bifurcations. A Hopf bifurcation is a critical point where the stability switches and a limit cycle arises. More precisely, it is a local bifurcation around an equilibrium point, which occurs when a simple pair of complex conjugate eigenvalues cross the imaginary axis as certain parameters are varied. We can classify Hopf bifurcations as supercritical or subcritical, according to whether the nonlinearity is destabilising or stabilising respectively. The Hopf bifurcation of autonomous systems is widely described in literature. The extensive list of references connecting with this problem is for instance presented in [1, 2, 3, 4, 5].

Based on the center manifold theorem and the normal form theory, Hassard, Kazarinoff and Wan [2] gave a comprehensive treatment of Hopf bifurcation theorem and its generalizations and applications, and developed a set of bifurcation formulae for the parameters $\mu_{2}, \tau_{2}$ and $\beta_{2}$. These formulae can be used to determine the direction of the bifurcation, the period, the amplitude and the stability of bifurcating limit cycles. However, the calculations for these parameters involve so many high order partial derivatives of the nonlinear functions in the real canonical system, which lead to a heavy computational load.

E-mail address: sangbo_76@163.com.

Received February 20, 2019; Accepted July 4, 2019.

(C)2019 Journal of Nonlinear Functional Analysis 
Even for some simple 3-D quadratic systems, for example, Rössler model (see [6, Conjecture 5]), the computational load is still very heavy. For this reason, we present the concise expressions of $\mu_{2}, \tau_{2}, \beta_{2}$ for the general 3-D quadratic systems in Section 2. The computation is now based on the original formulae [2], the complex canonical form, the chain rule, the complex arithmetic and the nature of quadratic systems. The use of complex canonical form is inspired by the previous work $[7,8,9,10]$ for 3-D systems, where the computation of Lyapunov coefficients (also known as focus quantities, focus values, etc.) was discussed. For the general multi-dimensional systems, Lyapunov coefficients can be used to determine the stabilities and the number of bifurcating limit cycles. The first Lyapunov coefficient (equivalent to $\beta_{2}$ ) was derived in [11] via projection methods. Chaos theory is a branch of mathematics that deals with nonlinear dynamical systems, which have a very sensitive dependence on their initial conditions. After the discovery of chaos in a 3-D weather model, i.e., the Lorenz system [12], the modelling and analysis of new chaotic systems have been extensively investigated. In particular, there has been a strong interest in the study of 3-D quadratic chaotic systems, see [13,14]. In fact, this class represents the minimal complexity in dimension and nonlinearity such that a system can display chaotic motions.

The analytical study of local bifurcations (Hopf bifurcations, pitchfork bifurcations, saddle-node bifurcations, zero-Hopf bifurcations, etc) in the chaotic systems can help to understand the formulating mechanism of strange attractors. Although many studies have been made on Hopf bifurcations of some special 3-D quadratic systems: Chen system [15, 16], economic chaotic systems [17, 18, 19], Lorenzlike systems [20, 21, 22], Lü system [15, 23], Maxwell-Bloch system [24], Rikitake system [25], Rössler system [6], Rucklidge system [26], Shimizu-Morioka system [27], $T$ system [28], Genesio-Tesi system $[29,30,31]$ and so on, a challenge still exists for producing a concise unified scheme on the Hopf bifurcation analysis. Furthermore, the results in [31] on Hopf bifurcation are incomplete due to a lack of consideration of the period, amplitude and analytical approximations of limit cycles; the statement of criticality is incorrect (This error was first discovered and corrected by Dias and Mello [29] with the projection method). This motivates us to derive a set of bifurcation formulae for the general family of 3-D quadratic systems (includes the quadratic family in [29]) independently, not based on the projection method. By the way, Cardin and Llibre [32] studied the transcritical and Zero-Hopf bifurcations of Genesio-Tesi system.

Consider a jerk equation

$$
\frac{d^{3} x}{d t^{3}}=f\left(x, \frac{d x}{d t}, \frac{d^{2} x}{d t^{2}}\right)
$$

with a nonlinear function $f$. The term "jerk" comes from the fact that in a mechanical system in which $x$ is the displacement, $\frac{d x}{d t}$ is the velocity, and $\frac{d^{2} x}{d t^{2}}$ is the acceleration, the quantity $\frac{d^{3} x}{d t^{3}}$ is called the "jerk", see [14, 33]. The assignments $x=x, y=\frac{d x}{d t}, z=\frac{d y}{d t}$ transform the equation into the following system

$$
\left\{\begin{array}{l}
\frac{d x}{d t}=y, \\
\frac{d y}{d t}=z \\
\frac{d z}{d t}=f(x, y, z),
\end{array}\right.
$$

which is called a jerk system. 
Inspired by the problem of determining conditions under which a nonlinear differential system presents chaotic behavior, Genesio and Tesi [30] introduced the following jerk equation

$$
\frac{d^{3} x}{d t^{3}}+a \frac{d^{2} x}{d t^{2}}+b \frac{d x}{d t}+c x-x^{2}=0
$$

where $a, b, c \in \mathbb{R}^{+}$. Let $x=x, y=\frac{d x}{d t}, z=\frac{d y}{d t}$. Then the equation can be transformed into

$$
\left\{\begin{array}{l}
\frac{d x}{d t}=y, \\
\frac{d y}{d t}=z, \\
\frac{d z}{d t}=-c x-b y-a z+x^{2},
\end{array}\right.
$$

which is known as the Genesio-Tesi system (also called the Genesio system). This jerk system serves as a paradigm for chaos since it captures many features of chaotic systems, see, for instance, [13, 34]. Since $c>0$, it has two equilibrium points : $O:=(0,0,0)$ and $G:=(c, 0,0)$.

1.1. Divergence. Let $V=X \frac{\partial}{\partial x}+Y \frac{\partial}{\partial y}+Z \frac{\partial}{\partial z}$ be the vector field of system (1.1). The divergence of the vector field $V$ is

$$
X_{x}+Y_{y}+Z_{z}=-a
$$

which is negative since $a>0$. Denoting a typical element of phase-space volume by $\Gamma(t)$, we thus have a contraction of the form

$$
\Gamma(t)=\Gamma(0) e^{-a t}
$$

Hence the volume in phase-space shrinks exponentially fast. The dynamical system is dissipative and can have stable attractors.

1.2. Local analysis of the equilibrium $O$. The Jacobian matrix of the Genesio-Tesi system at $O$ is

$$
J_{O}:=\left(\begin{array}{ccc}
0 & 1 & 0 \\
0 & 0 & 1 \\
-c & -b & -a
\end{array}\right) .
$$

We now consider the characteristic polynomial of $J_{O}$, i.e.,

$$
\Phi_{O}(\lambda):=\lambda^{3}+s_{11} \lambda^{2}+s_{12} \lambda+s_{13},
$$

where $s_{11}=a, s_{12}=b$ and $s_{13}=c$. According to the Routh-Hurwitz criterion, see, for instance, [35, Theorem 6, pages 58-59], the equilibrium $O$ is asymptotically stable if $s_{11}>0, s_{12}>0, s_{13}>0$ and $s_{13}<s_{11} s_{12}$. Since we have assumed that $a, b, c \in \mathbb{R}^{+}$, it is asymptotically stable if $c<a b$.

Let $\Delta_{O}$ be the discriminant of $\Phi_{O}(\lambda)$, i.e.,

$$
\Delta_{O}=-4 a^{3} c+a^{2} b^{2}+18 a b c-4 b^{3}-27 c^{2} .
$$

If $\Delta_{O}>0$, then $\Phi_{O}(\lambda)$ has three distinct real roots. If $\Delta_{O}<0$, then $\Phi_{O}(\lambda)$ has three distinct roots: one is real and two form a complex conjugate pair. If $\Delta_{O}=0$, then $\Phi_{O}(\lambda)$ has only real roots, one of which occurs with multiplicity at least 2 .

Since $s_{11}=a>0, s_{12}=b>0, s_{13}=c>0$, it follows from [36, Theorem 5.15-5.16, page 132], [37, pages 181-182] and [38, page 2014] that: 
(i) If $\Delta_{O} \geq 0$, then all the roots of $\Phi_{O}(\lambda)$ are negative. In this case the equilibrium $O$ is a stable node.

(ii) If $\Delta_{O}<0$, then the real root of $\Phi_{O}(\lambda)$ is negative. In this case, if $s_{11} s_{12}-s_{13}=a b-c>0$ the equilibrium $O$ is a stable focus-node; if $s_{11} s_{12}-s_{13}=a b-c<0$ it is a saddle-focus; if $s_{11} s_{12}-s_{13}=$ $a b-c=0$, i.e., $c=a b$, then a Hopf bifurcation can occur. In fact, we can choose $c$ as a bifurcation parameter, that is, $s_{11}, s_{12}, s_{13}$ can be considered as function of $c$ with $s_{11}(c)=a, s_{12}(c)=b, s_{13}(c)=c$. For $c=a b$, we have $s_{11}(a b)=a \neq 0, s_{12}(a b)=b>0, s_{11}(a b) s_{12}(a b)-s_{13}(a b)=0$ and

$$
\left.\left[s_{11}(c) s_{12}(c)-s_{13}(c)\right]\right|_{c=a b} ^{\prime}=-1 \neq 0 .
$$

Thus all the conditions of the Hopf bifurcation are satisfied, see [39, Proposition, page 634-635] or [40, Theorem, page 252].

1.3. Local analysis of the equilibrium $G$. The Jacobian matrix of the Genesio-Tesi system at $G$ is

$$
J_{G}:=\left(\begin{array}{ccc}
0 & 1 & 0 \\
0 & 0 & 1 \\
c & -b & -a
\end{array}\right) .
$$

Let us consider the characteristic polynomial of $J_{G}$, i.e.,

$$
\Phi_{G}(\lambda):=\lambda^{3}+s_{21} \lambda^{2}+s_{22} \lambda+s_{23},
$$

where $s_{21}=a, s_{22}=b$ and $s_{23}=-c$. Since $s_{23}=-c<0$ and $s_{21} s_{22}-s_{23}=a b+c>0, G$ is always hyperbolic and unstable. Thus, the Hopf bifurcation cannot occur in the neighborhood of $G$.

Let $\Delta_{G}$ be the discriminant of $\Phi_{G}(\lambda)$, i.e.,

$$
\Delta_{G}=4 a^{3} c+a^{2} b^{2}-18 a b c-4 b^{3}-27 c^{2} .
$$

Since $s_{21}=a>0, s_{22}=b>0, s_{23}=-c<0$, it follows from [36, Theorem 5.15-5.16, page 132] and [38, page 2014] that:

(i) If $\Delta_{G} \geq 0$, then one real root of $\Phi_{G}(\lambda)$ is positive, the other real roots are negative. In this case $G$ is a saddle point.

(ii) If $\Delta_{G}<0$, then the real root $\lambda_{1}$ of $\Phi_{G}(\lambda)$ is positive. In this case the other two $\lambda_{2}, \lambda_{3}$ are complex conjugate roots; since $\lambda_{1}+\left(\lambda_{2}+\lambda_{3}\right)=\lambda_{1}+2 \Re\left(\lambda_{2}\right)=-s_{21}=-a<0$, we have $\Re\left(\lambda_{2}\right)<0$. Hence $G$ is a saddle-focus.

1.4. The structure of the rest part of this paper. We have discussed some basic properties of the Genesio-Tesi system in details, with the same restriction on the parameters as in [30], i.e., $a, b, c \in \mathbb{R}^{+}$. The rest of this paper is organized as follows. In order to facilitate the analysis of Hopf bifurcations in the general 3-D quadratic systems, we present a set of concise bifurcation formulae for $\mu_{2}, \tau_{2}$ and $\beta_{2}$ in Section 2. In Section 3, using these formulae, we study the qualitative properties of Hopf bifurcation in the Genesio-Tesi system. Due to the positiveness of parameters, the Hopf bifurcation can only occur at the origin when $c=a b$. By a direct computation, we determine the direction of the bifurcation, the period, amplitude and stability of bifurcating limit cycles. Dias and Mello [29] discussed the same system with the projection method, however, the period, amplitude of limit cycles and the routes to chaos were not taken into account. Thus their discussion is still incomplete. Due to the general-purpose formulae in Section 2, we can conclude the contributions of this section and the next section are independent 
and necessary. The last section is devoted to the verification of theoretical results by means of numerical simulations. The underlying mechanisms leading to chaos are also discussed with some numerical simulations.

\section{HOPF BIFURCATION FOR THREE-DIMENSIONAL QUADRATIC SYSTEMS}

Consider a class of 3-D autonomous systems

$$
\frac{d x}{d t}=f(x, \mu), x=\left(x_{1}, x_{2}, x_{3}\right)^{T} \in \mathbb{R}^{3}, \mu \in \mathscr{J} \subseteq \mathbb{R},
$$

where $x$ is the state variable and $\mu$ is the distinguished bifurcation parameter. In Section 2.1, following [2, 41, 42], we give a brief description on the Hopf bifurcation of system (2.1). In Section 2.2, for the general 3-D quadratic systems, we present a set of bifurcation formulae for $\mu_{2}, \tau_{2}$ and $\beta_{2}$. In contrast to the original version $[2,41]$, these formulae are more concise and convenient in handling the general 3-D quadratic systems. This improvement can help to study the Hopf bifurcations of many mathematically or physically important 3-D quadratic systems.

2.1. Hopf bifurcation theory of 3-D systems. According to [2, 41, 42], we have the following result.

Proposition 2.1. For system (2.1), we assume that the following hypotheses hold.

(H1) $f$ is sufficiently smooth in $x$ and $\mu$ in a neighborhood of $\left(x_{0}, \mu_{0}\right) \in \mathbb{R}^{3} \times \mathbb{R}$, where $x_{0}=x\left(\mu_{0}\right)$;

(H2) There is an isolated equilibrium point $x(\mu)$ of the system for values of $\mu \in \mathscr{J}$;

(H3) The Jacobian matrix $D_{x} f(x(\mu), \mu)$ has a pair of complex conjugate eigenvalues, $\lambda_{1,2}:=\alpha(\mu) \pm$ $i \omega(\mu)$, and a negative eigenvalue $\lambda_{3}:=d(\mu)<0$, with $\omega\left(\mu_{0}\right)=\omega_{0}>0, \alpha\left(\mu_{0}\right)=0, \alpha^{\prime}\left(\mu_{0}\right) \neq 0$.

Generically, the system has a family of small limit cycles. In particular, there is a real number $\mu_{2}$ such that if $\mu_{2} \neq 0$, the limit cycle can be approximated by

$$
x=p(t ; \mu)=x_{0}+\sqrt{\frac{\mu-\mu_{0}}{\mu_{2}}} \Re\left(e^{\frac{2 \pi i t}{T(\mu)}} u_{1}\right)+\mathscr{O}\left(\mu-\mu_{0}\right),
$$

where

$$
T(\mu)=\frac{2 \pi}{\omega_{0}}\left(1+\frac{\tau_{2}}{\mu_{2}}\left(\mu-\mu_{0}\right)\right)+\mathscr{O}\left(\left(\mu-\mu_{0}\right)^{2}\right)
$$

and $u_{1}$ is the eigenvector of the Jacobian matrix $D_{x} f\left(x_{0}, \mu_{0}\right)$ corresponding to $\lambda_{1}\left(\mu_{0}\right)$, with its first nonvanishing component being 1. If $\mu_{2}>0$, the limit cycles exist for $\mu>\mu_{0}$, and if $\mu_{2}<0$, the limit cycles exist for $\mu<\mu_{0}$. Moreover, if $\beta_{2}:=-2 \alpha^{\prime}\left(\mu_{0}\right) \mu_{2}<0$, the limit cycles are orbitally asymptotically stable, while if $\beta_{2}>0$ they are unstable.

The expressions for $\mu_{2}, \tau_{2}$ and $\beta_{2}$ for finite-dimensional systems were given in [2], together with the long procedure required to obtain them. In Proposition 2.1, if $p(t ; \mu)=\left(x_{1}(t ; \mu), x_{2}(t ; \mu), x_{3}(t ; \mu)\right)^{T}$, $x_{0}=\left(x_{10}, x_{20}, x_{30}\right)^{T}$ and $u_{1}=\left(u_{11}+i u_{12}, u_{21}+i u_{22}, u_{31}+i u_{32}\right)^{T}$ with $u_{i j} \in \mathbb{R}$, then the limit cycle (2.2) can be written in component form as

$$
\left\{\begin{array}{l}
x_{1}(t ; \mu)=x_{10}+R(\mu)\left(\cos \left(\omega_{0} t\right) u_{11}-\sin \left(\omega_{0} t\right) u_{12}\right)+\mathscr{O}\left(\mu-\mu_{0}\right), \\
x_{2}(t ; \mu)=x_{20}+R(\mu)\left(\cos \left(\omega_{0} t\right) u_{21}-\sin \left(\omega_{0} t\right) u_{22}\right)+\mathscr{O}\left(\mu-\mu_{0}\right), \\
x_{3}(t ; \mu)=x_{30}+R(\mu)\left(\cos \left(\omega_{0} t\right) u_{31}-\sin \left(\omega_{0} t\right) u_{32}\right)+\mathscr{O}\left(\mu-\mu_{0}\right),
\end{array}\right.
$$

where

$$
R(\mu):=\sqrt{\frac{\mu-\mu_{0}}{\mu_{2}}}
$$


is the approximate amplitude.

After outlining some basic results of Hopf bifurcation in 3-D systems, we are now in a position to develop our method based on "complex canonical form". In [9], we have taken the advantage of it without giving a name. Thus, it is necessary to formulate this concept in the setting of Proposition 2.1.

Let $P=\left(v_{1}, \overline{v_{1}}, r_{3}\right)$, where $v_{1}:=\frac{1}{2} u_{1}$ with $u_{1}$ coming from Proposition 2.1, and $r_{3}$ be the eigenvector of $D_{x} f\left(x_{0}, \mu_{0}\right)$ corresponding to $\lambda_{3}\left(\mu_{0}\right):=d$. Applying the transformation $x=P z+x_{0}$, we see that system (2.1) $\left.\right|_{\mu=\mu_{0}}$ becomes

$$
\frac{d z}{d t}=A(z), z=\left(z_{1}, z_{2}, z_{3}\right)^{T},
$$

whose Jacobian matrix evaluated at the origin is

$$
D A(0)=\left(\begin{array}{ccc}
i \omega_{0} & 0 & 0 \\
0 & -i \omega_{0} & 0 \\
0 & 0 & d
\end{array}\right)
$$

(2.6) is called the complex canonical form of system (2.1) for $\mu=\mu_{0}$. Let

$$
Q=\left(\begin{array}{ccc}
1 & i & 0 \\
1 & -i & 0 \\
0 & 0 & 1
\end{array}\right) \text {. }
$$

By using the transformation $z=Q y$, system (2.6) can be transformed into

$$
\frac{d y}{d t}=F(y), y=\left(y_{1}, y_{2}, y_{3}\right)^{T} \in \mathbb{R}^{3},
$$

whose Jacobian matrix evaluated at the origin is

$$
D F(0)=\left(\begin{array}{ccc}
0 & -\omega_{0} & 0 \\
\omega_{0} & 0 & 0 \\
0 & 0 & d
\end{array}\right) .
$$

Accordingly, (2.7) can be called the real canonical form of system (2.1) for $\mu=\mu_{0}$. In consequence, by using the transformation $x=P Q y+x_{0}$, system (2.1) $\left.\right|_{\mu=\mu_{0}}$ can be transformed into system (2.7). It should be noted that

$$
P Q=\left(v_{1}+\overline{v_{1}}, \mathrm{i}\left(v_{1}-\overline{v_{1}}\right), r_{3}\right)=\left(2 \mathfrak{R}\left(v_{1}\right),-2 \mathfrak{I}\left(v_{1}\right), r_{3}\right) ;
$$

according to the facts $v_{1}=\frac{1}{2} u_{1}$, and the first non-vanishing component of $v_{1}+\overline{v_{1}}$ is 1 , i.e., $v_{1}+\overline{v_{1}}$ is a normalized vector in the sense of [2, Chapter 2, page 87].

For systems (2.6) and (2.7), we set $A=\left(A_{1}, A_{2}, A_{3}\right)^{T}$ and $F=\left(F^{1}, F^{2}, F^{3}\right)^{T}$, respectively. According to the transformation $z=Q y$ between these systems, we have

$$
A_{1}(Q y)=F^{1}(y)+\text { i } F^{2}(y), A_{2}(Q y)=F^{1}(y)-\text { i } F^{2}(y), A_{3}(Q y)=F^{3}(y),
$$

where

$$
A_{1}=\overline{A_{2}}, \quad A_{3} \in \mathbb{R}
$$


2.2. Hopf bifurcation formulae for quadratic systems. Instead of presenting results for general systems, we restrict our attention to the quadratic systems. From now on, we shall assume that system (2.1) is a 3-D quadratic system. Based on the original formulae of Hassard et al. [2], we now use the chain rule and complex arithmetic to obtain the explicit forms of $\mu_{2}, \tau_{2}, \beta_{2}$, which are in terms of second-order partial derivatives of the components of $A$. For convenience, let us introduce the abbreviations

$$
m_{k s j}=\frac{\partial^{2} A_{k}}{\partial z_{s} \partial z_{j}}, \quad k, s, j=1,2,3 .
$$

According to [2], there are many intermediate terms involved in a reduction to the Poincaré normal form, i.e., $g_{11}, g_{02}, g_{20}, G_{21}, h_{11}^{1}, h_{20}^{1}, w_{11}^{1}, w_{20}^{1}, G_{110}^{1}, G_{101}^{1}, g_{21}, c_{1}(0)$ and $\overline{c_{1}(0)}$. Using the chain rule for partial differentiation, we get

$$
\begin{aligned}
& g_{11}=\frac{1}{4}\left[F_{y_{1}, y_{1}}^{1}+F_{y_{2}, y_{2}}^{1}+i\left(F_{y_{1}, y_{1}}^{2}+F_{y_{2}, y_{2}}^{2}\right)\right] \\
& =\frac{1}{4}\left[\frac{\partial^{2} A_{1}(Q y)}{\partial y_{1}^{2}}+\frac{\partial^{2} A_{1}(Q y)}{\partial y_{2}^{2}}\right] \\
& =m_{112} \text {, } \\
& g_{02}=\frac{1}{4}\left[F_{y_{1}, y_{1}}^{1}-F_{y_{2}, y_{2}}^{1}-2 F_{y_{1}, y_{2}}^{2}+i\left(F_{y_{1}, y_{1}}^{2}-F_{y_{2}, y_{2}}^{2}+2 F_{y_{1}, y_{2}}^{1}\right)\right] \\
& =\frac{1}{4}\left[\frac{\partial^{2} A_{1}(Q y)}{\partial y_{1}^{2}}-\frac{\partial^{2} A_{1}(Q y)}{\partial y_{2}^{2}}+2 i \frac{\partial^{2} A_{1}(Q y)}{\partial y_{1} \partial y_{2}}\right] \\
& =m_{122} \text {, } \\
& g_{20}=\frac{1}{4}\left[F_{y_{1}, y_{1}}^{1}-F_{y_{2}, y_{2}}^{1}+2 F_{y_{1}, y_{2}}^{2}+\mathrm{i}\left(F_{y_{1}, y_{1}}^{2}-F_{y_{2}, y_{2}}^{2}-2 F_{y_{1}, y_{2}}^{1}\right)\right] \\
& =\frac{1}{4}\left[\frac{\partial^{2} A_{1}(Q y)}{\partial y_{1}^{2}}-\frac{\partial^{2} A_{1}(Q y)}{\partial y_{2}^{2}}-2 i \frac{\partial^{2} A_{1}(Q y)}{\partial y_{1} \partial y_{2}}\right] \\
& =m_{111} \text {, } \\
& G_{21}=0 \text {, } \\
& h_{11}^{1}=\frac{1}{4}\left[F_{y_{1}, y_{1}}^{3}+F_{y_{2}, y_{2}}^{3}\right] \\
& =\frac{1}{4}\left[\frac{\partial^{2} A_{3}(Q y)}{\partial y_{1}^{2}}+\frac{\partial^{2} A_{3}(Q y)}{\partial y_{2}^{2}}\right] \\
& =m_{312} \text {, } \\
& h_{20}^{1}=\frac{1}{4}\left[F_{y_{1}, y_{1}}^{3}-F_{y_{2}, y_{2}}^{3}-2 \mathrm{i} F_{y_{1}, y_{2}}^{3}\right] \\
& =\frac{1}{4}\left[\frac{\partial^{2} A_{3}(Q y)}{\partial y_{1}^{2}}-\frac{\partial^{2} A_{3}(Q y)}{\partial y_{2}^{2}}-2 i \frac{\partial^{2} A_{3}(Q y)}{\partial y_{1} \partial y_{2}}\right] \\
& =m_{311} \text {, } \\
& w_{11}^{1}=-\frac{h_{11}^{1}}{d} \\
& =-\frac{m_{312}}{d}, \\
& w_{20}^{1}=-\frac{h_{20}^{1}}{d-2 \omega_{0} \dot{i}}
\end{aligned}
$$




$$
\begin{aligned}
& =-\frac{m_{311}}{d-2 \omega_{0} i}, \\
G_{110}^{1} & =\frac{1}{2}\left[F_{y_{1}, y_{3}}^{1}+F_{y_{2}, y_{3}}^{2}+i\left(F_{y_{1}, y_{3}}^{2}-F_{y_{2}, y_{3}}^{1}\right)\right] \\
& =\frac{1}{2}\left[\frac{\partial^{2} A_{1}(Q y)}{\partial y_{1} \partial y_{3}}-i \frac{\partial^{2} A_{1}(Q y)}{\partial y_{2} \partial y_{3}}\right], \\
& =m_{113}, \\
G_{101}^{1} & =\frac{1}{2}\left[F_{y_{1}, y_{3}}^{1}-F_{y_{2}, y_{3}}^{2}+i\left(F_{y_{1}, y_{3}}^{2}+F_{y_{2}, y_{3}}^{1}\right)\right] \\
& =\frac{1}{2}\left[\frac{\partial^{2} A_{1}(Q y)}{\partial y_{1} \partial y_{3}}+i \frac{\partial^{2} A_{1}(Q y)}{\partial y_{2} \partial y_{3}}\right], \\
& =m_{123} .
\end{aligned}
$$

Thus,

$$
\begin{gathered}
g_{21}=G_{21}+2 G_{110}^{1} w_{11}^{1}+G_{101}^{1} w_{20}^{1} \\
=2 G_{110}^{1} w_{11}^{1}+G_{101}^{1} w_{20}^{1} \\
=-\frac{2 m_{113} m_{312}}{d}-\frac{m_{123} m_{311}}{d-2 \omega_{0} i}, \\
c_{1}(0)=\frac{i}{2 \omega_{0}}\left(g_{20} g_{11}-2\left|g_{11}\right|^{2}-\frac{1}{3}\left|g_{02}\right|^{2}\right)+\frac{g_{21}}{2} \\
=\frac{i}{2 \omega_{0}}\left(m_{111} m_{112}-2 m_{112} m_{212}-\frac{1}{3} m_{122} m_{211}\right)-\frac{m_{113} m_{312}}{d} \\
-\frac{m_{123} m_{311}}{2\left(d-2 \omega_{0} i\right)}, \\
\frac{-i}{c_{1}(0)}=\frac{-}{2 \omega_{0}}\left(m_{222} m_{212}-2 m_{112} m_{212}-\frac{1}{3} m_{122} m_{211}\right) \\
-\frac{m_{223} m_{312}}{d}-\frac{m_{213} m_{322}}{2\left(d+2 \omega_{0} i\right)} .
\end{gathered}
$$

It should be noted that the last equation follows from (2.8), (2.9) and (2.10).

Next, by applying the bifurcation formulae of Hassard et al. [2, page 90] and using the above simplifications for intermediate terms, we have the following result.

Theorem 2.2. Assume that system (2.1) is a 3-D quadratic system and all the conditions of Proposition 2.1 are satisfied. Then the bifurcation formulae can be expressed as follows

$$
\begin{aligned}
\mu_{2} & =-\frac{\mathfrak{R}\left(c_{1}(0)\right)}{\alpha^{\prime}\left(\mu_{0}\right)}, \\
\tau_{2} & =-\frac{\mathfrak{I}\left(c_{1}(0)\right)}{\omega_{0}}-\mu_{2} \frac{\omega^{\prime}\left(\mu_{0}\right)}{\omega_{0}}, \\
\beta_{2} & =2 \Re\left(c_{1}(0)\right),
\end{aligned}
$$

where 


$$
\begin{aligned}
\mathfrak{R}\left(c_{1}(0)\right)= & \frac{c_{1}(0)+\overline{c_{1}(0)}}{2} \\
= & -\frac{m_{312}\left(m_{113}+m_{223}\right)}{2 d}+\frac{i\left(m_{111} m_{112}-m_{222} m_{212}\right)}{4 \omega_{0}}-\frac{m_{213} m_{322}}{4\left(d+2 i \omega_{0}\right)} \\
& +\frac{m_{123} m_{311}}{4\left(2 i \omega_{0}-d\right)}, \\
\mathfrak{I}\left(c_{1}(0)\right)= & \frac{c_{1}(0)-\overline{c_{1}(0)}}{2 i} \\
= & \frac{i m_{312}\left(m_{113}-m_{223}\right)}{2 d}+\frac{3 m_{111} m_{112}-12 m_{112} m_{212}-2 m_{122} m_{211}+3 m_{222} m_{212}}{12 \omega_{0}} \\
& -\frac{i m_{213} m_{322}}{4\left(d+2 i \omega_{0}\right)}-\frac{i m_{123} m_{311}}{4\left(2 i \omega_{0}-d\right)} .
\end{aligned}
$$

Proof. Using (2.11) and (2.12), we get (2.16) and (2.17). According to the general formulae [2, page 90], we obtain (2.13), (2.14) and (2.15).

2.3. Remarks and discussions. For a class of quadratic 3-D systems, we developed a set of explicit expressions for $\mu_{2}, \tau_{2}, \beta_{2}$ in terms of second-order partial derivatives of the functions $A_{1}, A_{2}$ and $A_{3}$. By means of these bifurcation formulae, we can determine the direction of bifurcation, the period, amplitude and stability of bifurcating limit cycles at the critical value of the bifurcation parameter. However, without the previous simplifications for the intermediate terms, the computational load would become very heavy due to the tedious substitutions involved. This partially explains the fact [2]: the algorithm of Hassard et al. is not effective for handling the problems beyond a certain complexity. With some necessary modifications, the simplifications for intermediate terms can be generalized to multi-dimensional systems.

\section{A Hopf BIFURCATION IN THE GenESIO-Tesi SySTEM}

In general, the study of Hopf bifurcation includes the existence and the properties such as the direction of bifurcation and the stability of bifurcating limit cycles. In practice, however, it is more difficult to determine the properties of a Hopf bifurcation than to find the existence of a Hopf bifurcation. In Section 1.2 we showed the existence of a Hopf bifurcation in the Genesio-Tesi system. Based on the formulae obtained in Section 2, we next explore some properties: the direction of bifurcation, the period, the amplitude, and the stability of bifurcating limit cycles at the critical value $c=c_{0}:=a b$. For the sake of convenience, we set $\Psi(\lambda ; c):=\Phi_{O}(\lambda)$, where $\Phi_{O}(\lambda)$ can be seen in Section 1.2. When $c=c_{0}:=a b$, $\Psi(\lambda ; c)$ has a simple pair of purely imaginary roots: $\lambda_{1,2}\left(c_{0}\right)= \pm i \sqrt{b}$ and a negative root $\lambda_{3}\left(c_{0}\right)=-a$. Let $\lambda_{1}(c)=\alpha(c)+i \omega(c)$ be the continuation of $\lambda_{1}\left(c_{0}\right)$, with $c$ near $c_{0}$. By a direct computation, we can check that

$$
\begin{aligned}
& \alpha^{\prime}\left(c_{0}\right)=-\left.\mathfrak{R}\left(\frac{\Psi_{c}}{\Psi_{\lambda}}\right)\right|_{\lambda=\lambda_{1}\left(c_{0}\right), c=c_{0}}=\frac{1}{2 a^{2}+2 b}>0, \\
& \omega^{\prime}\left(c_{0}\right)=-\left.\mathfrak{I}\left(\frac{\Psi_{c}}{\Psi_{\lambda}}\right)\right|_{\lambda=\lambda_{1}\left(c_{0}\right), c=c_{0}}=\frac{a}{2 \sqrt{b}\left(a^{2}+b\right)}>0 .
\end{aligned}
$$


By introducing the transformation

$$
\left\{\begin{array}{l}
x=\frac{1}{2} z_{1}+\frac{1}{2} z_{2}+\frac{z_{3}}{a^{2}} \\
y=\frac{i}{2} \sqrt{b} z_{1}-\frac{i}{2} \sqrt{b} z_{2}-\frac{z_{3}}{a} \\
z=-\frac{1}{2} b z_{1}-\frac{1}{2} b z_{2}+z_{3}
\end{array}\right.
$$

system $(1.1)_{c=c_{0}}$ can be transformed into

$$
\left\{\begin{aligned}
\frac{d z_{1}}{d t}= & i \sqrt{b} z_{1}-\frac{i z_{3}^{2}}{\sqrt{b} a^{4}(i \sqrt{b}+a)}-\frac{i z_{2} z_{3}}{\sqrt{b} a^{2}(i \sqrt{b}+a)} \\
& -\frac{i z_{2}^{2}}{4 \sqrt{b}(i \sqrt{b}+a)}-\frac{i z_{1} z_{3}}{\sqrt{b} a^{2}(i \sqrt{b}+a)}-\frac{i z_{1} z_{2}}{2 \sqrt{b}(i \sqrt{b}+a)}-\frac{i z_{1}^{2}}{4 \sqrt{b}(i \sqrt{b}+a)} \\
:= & A_{1}\left(z_{1}, z_{2}, z_{3}\right), \\
\frac{d z_{2}}{d t}= & -i \sqrt{b} z_{2}+\frac{i z_{3}^{2}}{\sqrt{b} a^{4}(-i \sqrt{b}+a)}+\frac{i z_{2} z_{3}}{\sqrt{b} a^{2}(-i \sqrt{b}+a)} \\
& +\frac{i z_{2}^{2}}{4 \sqrt{b}(-i \sqrt{b}+a)}+\frac{i z_{1} z_{3}}{\sqrt{b} a^{2}(-i \sqrt{b}+a)}+\frac{i z_{1} z_{2}}{2 \sqrt{b}(-i \sqrt{b}+a)}+\frac{i z_{1}^{2}}{4 \sqrt{b}(-i \sqrt{b}+a)} \\
:= & A_{2}\left(z_{1}, z_{2}, z_{3}\right), \\
\frac{d z_{3}}{d t}= & -a z_{3}+\frac{z_{3}^{2}}{a^{2}\left(a^{2}+b\right)}+\frac{z_{2} z_{3}}{a^{2}+b}+\frac{a^{2} z_{2}^{2}}{4\left(a^{2}+b\right)}+\frac{z_{1} z_{3}}{a^{2}+b}+\frac{a^{2} z_{1} z_{2}}{2\left(a^{2}+b\right)}+\frac{a^{2} z_{1}^{2}}{4\left(a^{2}+b\right)} \\
:= & A_{3}\left(z_{1}, z_{2}, z_{3}\right) .
\end{aligned}\right.
$$

In the setting of Proposition 2.1, we have $\mu=c, \mu_{0}=c_{0}=a b$ and $\omega_{0}=\sqrt{b}, d=-a$. According to (2.10), (2.16) and (2.17), we get

$$
\begin{aligned}
\mathfrak{R}\left(c_{1}(0)\right) & =\frac{-a^{2}-8 b}{4\left(a^{2}+4 b\right)\left(a^{2}+b\right) b a}, \\
\mathfrak{I}\left(c_{1}(0)\right) & =\frac{-5 a^{2}-26 b}{12\left(a^{2}+4 b\right) b^{3 / 2}\left(a^{2}+b\right)} .
\end{aligned}
$$

Furthermore, in view of (2.13), (2.14), (2.15), (3.1), (3.2), (3.4), (3.5), and $a, b \in \mathbb{R}^{+}$, we get

$$
\begin{aligned}
\mu_{2} & =\frac{a^{2}+8 b}{2 a b\left(a^{2}+4 b\right)}>0, \\
\tau_{2} & =\frac{1}{6 b^{2}\left(a^{2}+4 b\right)}>0, \\
\beta_{2} & =\frac{-a^{2}-8 b}{2\left(a^{2}+4 b\right)\left(a^{2}+b\right) b a}<0 .
\end{aligned}
$$

Let $u_{1}=\left(u_{11}+\mathbf{i} u_{12}, u_{21}+\mathbf{i} u_{22}, u_{31}+\mathbf{i} u_{32}\right)^{T}=(1, \sqrt{b} \dot{i},-b)^{T}$ with $u_{i j} \in \mathbb{R}$, where $u_{1}$ is the eigenvector of the Jacobian matrix of the system $(1.1)_{c=a b}$, evaluated at the origin, corresponding to the eigenvalue $\lambda_{1}\left(c_{0}\right)=i \sqrt{b}$. According to (2.3), (2.4) and (2.5), the limit cycle arising from the Hopf bifurcation can 
be written in component form as

$$
\left\{\begin{array}{l}
x(t ; c)=R(c) \cos (\sqrt{b} t)+\mathscr{O}(c-a b) \\
y(t ; c)=-\sqrt{b} R(c) \sin (\sqrt{b} t)+\mathscr{O}(c-a b) \\
z(t ; c)=-b R(c) \cos (\sqrt{b} t)+\mathscr{O}(c-a b)
\end{array}\right.
$$

where

$$
R(c)=\sqrt{\frac{2(c-a b) a b\left(a^{2}+4 b\right)}{a^{2}+8 b}}
$$

is approximate amplitude for $0<c-a b \ll 1$ and the approximate period is

$$
T(c)=\frac{2 \pi}{\sqrt{b}}\left(1+\frac{(c-a b) a}{3\left(a^{2}+8 b\right) b}\right)+\mathscr{O}\left((c-a b)^{2}\right)>\frac{2 \pi}{\sqrt{b}},
$$

where $0<c-a b \ll 1$.

Based on the above discussion, we have the following results.

Theorem 3.1. For the Genesio-Tesi system, the following statements hold.

(i) The Hopf bifurcation occurs at the origin when $c=a b$ is supercritical, i.e., the small limit cycles, which exist for $0<c-a b \ll 1$, are asymptotically stable.

(ii) The period of the bifurcating limit cycle increases with respect to $c$, where $c: 0<c-a b \ll 1$.

Proof. In view of the existence of Hopf bifurcation in Section 1.2, we only require the signs of $\mu_{2}, \tau_{2}$ and $\beta_{2}$ to prove the theorem. From (3.6), (3.7) and (3.8), we can conclude from Proposition 2.1 the desired conclusion immediatley.

Remark 3.2. The conclusion (i) was also obtained by Dias and Mello [29] with a different method (the projection method). However, the conclusion (ii) was not presented in [29].

\section{NUMERICAL SIMULATION ON HOPF BIFURCATIONS AND CHAOS}

In order to verify the analytical results, we carry out numerical simulations (based on the fourth-order Runge-Kutta method, with time step size 0.001 ) for some special cases of the Genesio-Tesi system, with the same initial conditions: $x(0)=y(0)=z(0)=0.1$. We plot respectively the trajectories for the cases $(a, b, c)=(1,1,0.9)$ and $(a, b, c)=(1,1,1.1)$. Since $a=b=1$, the critical value of $c$ is $c=1$ at which a Hopf bifurcation occurs. The origin is an asymptotically stable equilibrium point for $c<1$ and an unstable equilibrium point for $c>1$ with an asymptotically stable limit cycle for $c>1$. The Hopf bifurcation occurs at the origin when $c=1$ is supercritical, see Fig. 1.

Finally, we turn our attention to chaos in system (1.1). In the following discussion, we always choose $a=1, b=7$, the initial conditions $x(0)=y(0)=z(0)=0.5$ and vary $c$. According to Section 3, a supercritical Hopf bifurcation occurs at $c=7$, see Fig. 2, which are bifurcation diagrams of the successive maxima of $x$ as a function of $c$ in the ranges $[6,12]$ and $[12,15.5]$. It reveals the following route to chaos: stable equilibrium, limit cycle and the period doubling of the limit cycles. The largest Lyapunov exponents as a function of $c$ is plotted in Fig. 3 for $c \in[6,15.5]$, which is consistent with the bifurcation diagrams. Several periodic windows can be found in the chaotic regions, for example, $[14.31,14.33],[14.92,14.93],[15.17,15.22]$. See the enlarged views of bifurcation diagrams from Figure 2, i.e., Fig. 4, from which saddle-node bifurcations (SN), period doubling bifurcations (PD) and 


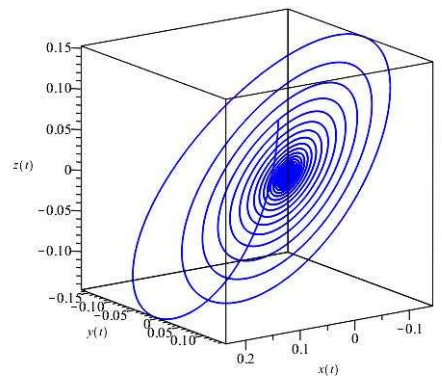

(a) $a=b=1, c=0.9$

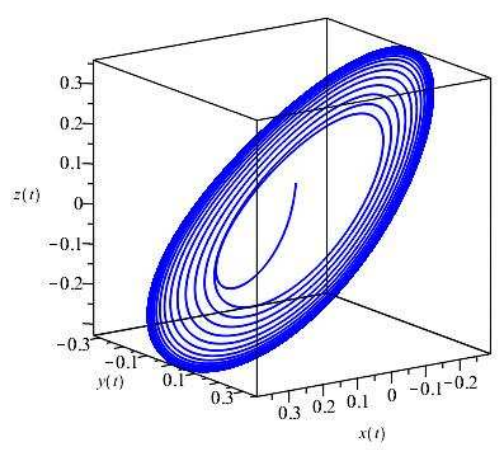

(b) $a=b=1, c=1.1$

Figure 1. (a) A trajectory of the Genesio-Tesi system spirals toward the origin, which is a stable equilibrium; (b) In the neighborhood of the origin, a trajectory of the GenesioTesi system spirals toward a stable limit cycle.

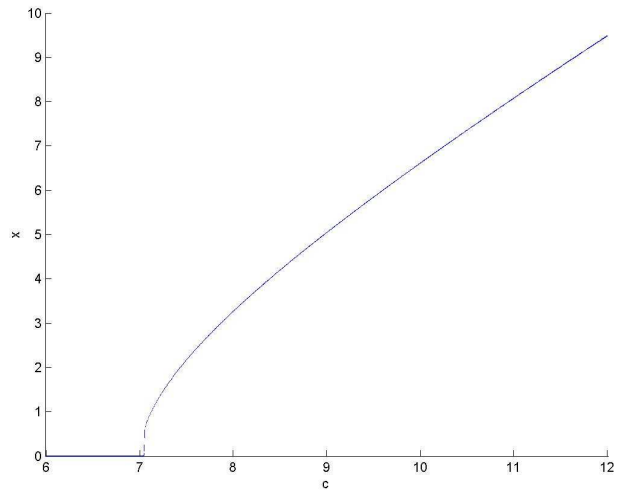

(a)

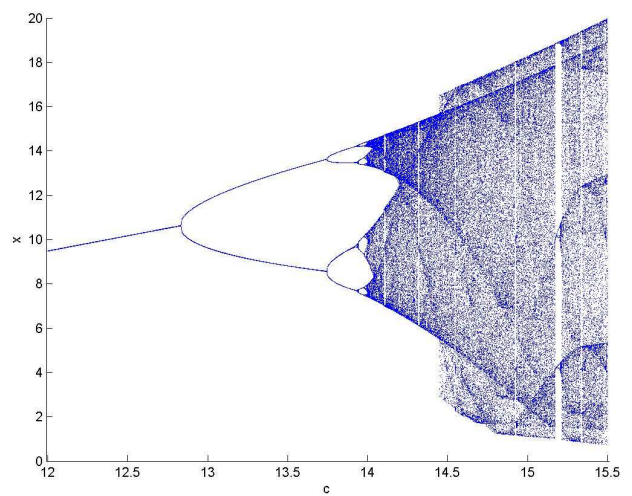

(b)

FigURE 2. (a) Bifurcation diagram for $c \in[6,12]$; (b) Bifurcation diagram for $c \in[12,15.5]$.

interior crises (IC) can be found. From Fig. 2, we can conclude that the interior crisis can also be found around $c=14.5$, and the boundary crisis can be found around 15.5.

TABLE 1. Lyapunov exponents

\begin{tabular}{c|c|c|c|c|c}
\hline$c$ & $L_{1}$ & $L_{2}$ & $L_{3}$ & Lyapunov Dimension & Dynamics \\
\hline 14.2 & 0.1433 & 0.0003 & -1.1436 & 2.1255 & Chaos \\
14.6 & 0.1971 & -0.0002 & -1.1969 & 2.1645 & Chaos \\
\hline
\end{tabular}

For the chaotic attractors in Fig. 5 with $c=14.2,14.6$, the Poincare maps on the plane $z=0$ and the Lyapunov exponents are depicted in Fig. 6 and Table 1, respectively. 


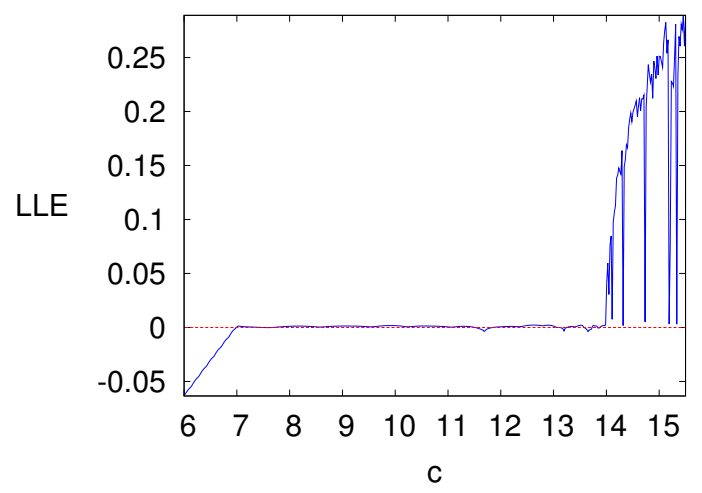

FIGURE 3

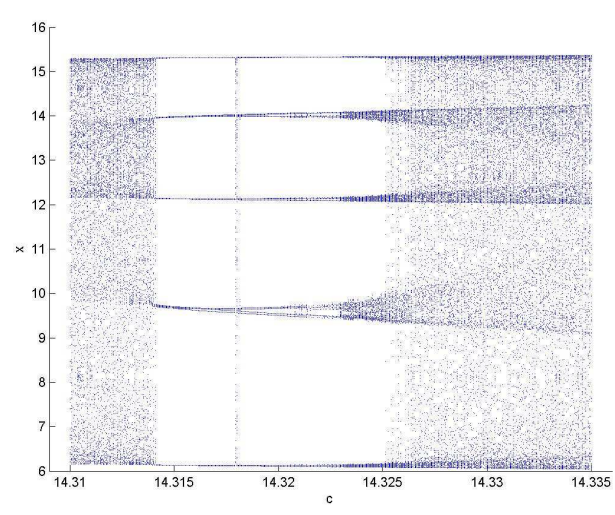

(a)

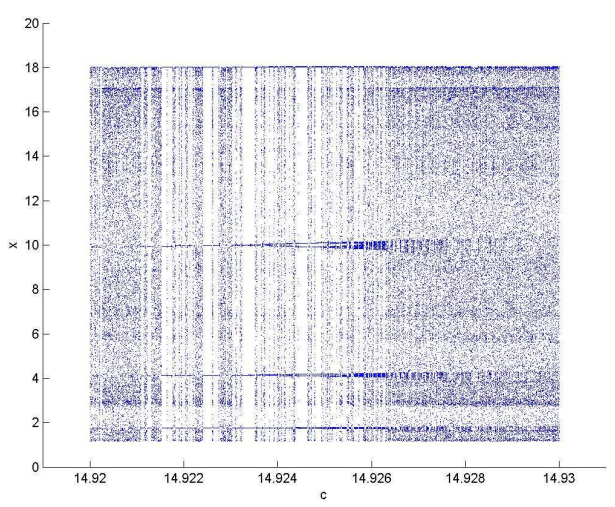

(b)

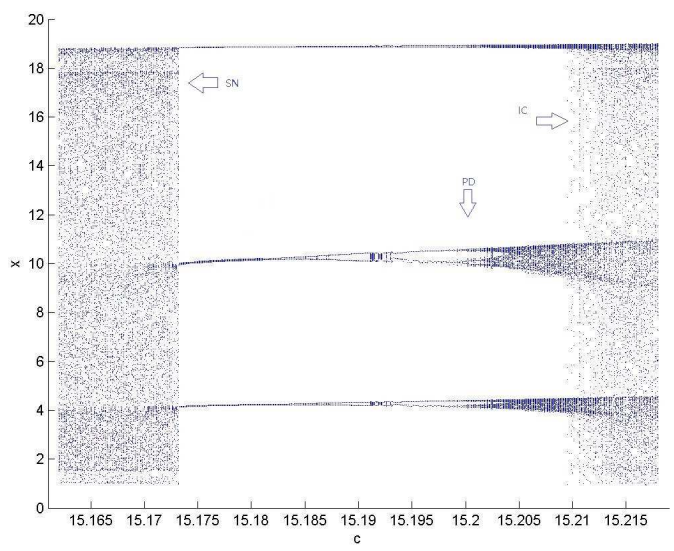

(c)

FIGURE 4. Periodic windows near $c=14.31,14.92,15.17$, respectively.

\section{Funding}

This paper was supported by Shandong Provincial Natural Science Foundation, China (ZR2018MA025). 


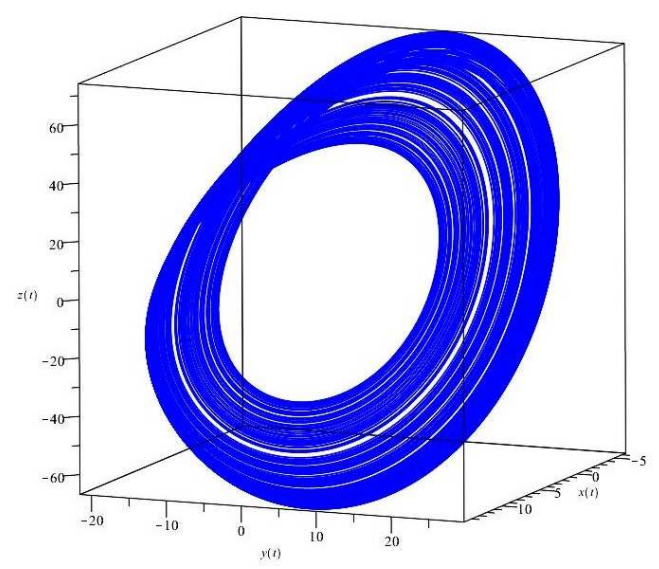

(a)

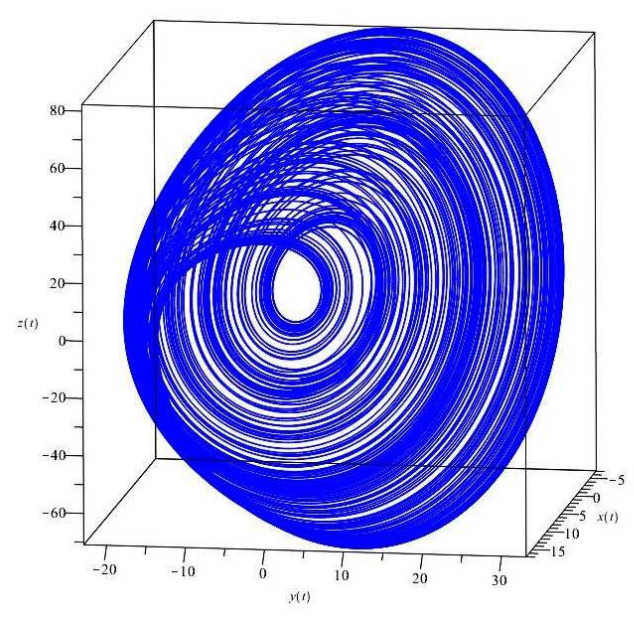

(b)

FIGURE 5. Chaotic attractors for $c=14.2,14.6$, respectively.

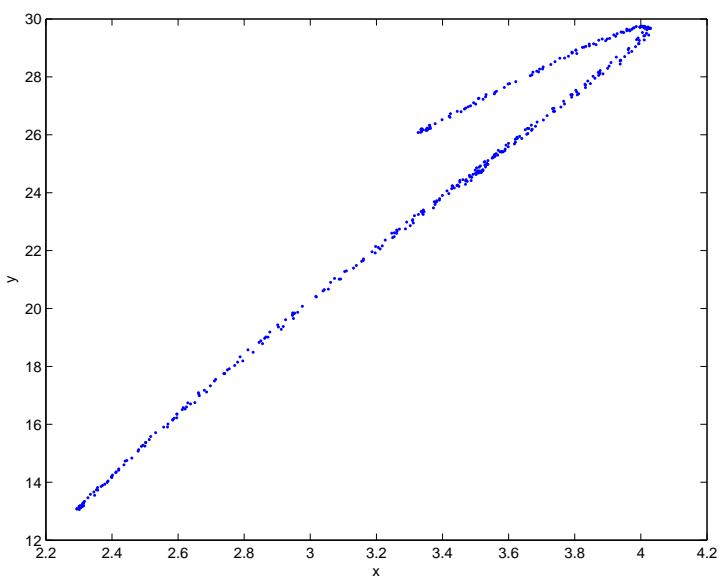

(a) $c=14.2$

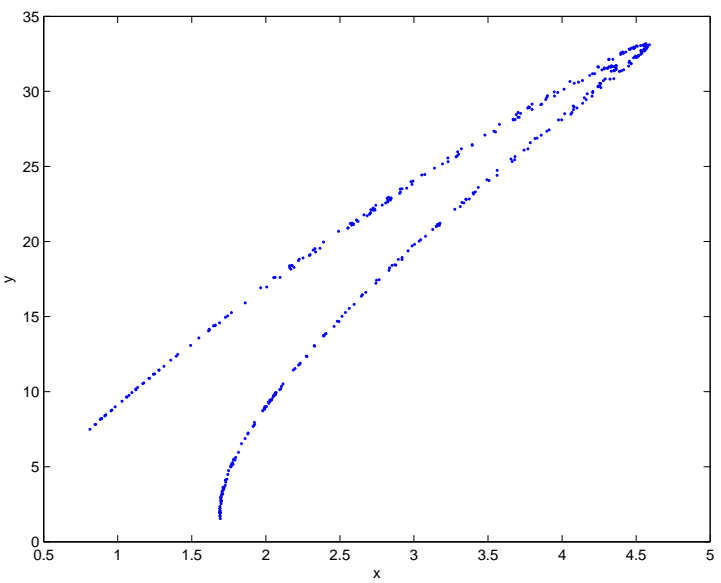

(b) $c=14.6$

FIGURE 6. Poincaré maps on $z=0$

\section{REFERENCES}

[1] S.N. Chow, J.K. Hale, Methods of Bifurcation Theory, Springer Verlag, New York, 1982.

[2] B.D. Hassard, N.D. Kazarinoff, Y.H. Wan, Theory and Applications of Hopf Bifurcation, Cambridge University Press, Cambridge, 1981.

[3] G. Iooss, D.D. Joseph, Elementary Stability and Bifurcation Theory, Undergraduate Texts in Mathematics, Springer Verlag, New York, 1990.

[4] J.E. Marsden, M. McCracken, The Hopf Bifurcation and Its Applications, Applied Mathematical Sciences, Vol. 19, Springer Verlag, New York, 1976.

[5] P. Yu, W. Lin, Complex dynamics in biological systems arising from multiple limit cycle bifurcation, J. Biological Dyn. 10 (2016), 263-285.

[6] R. Barrio, F. Blesa, A. Dena, S. Serrano, Qualitative and numerical analysis of the Rössler model: Bifurcations of equilibria, Comput. Math. Appl. 62 (2011), 4140-4150. 
[7] V.F. Edneral, A. Mahdi, V.G. Romanovski, D.S. Shafer, The center problem on a center manifold in $R^{3}$, Nonlinear Anal. 75 (2012), 2614-2622.

[8] V.G. Romanovski, D.S. Shafer, The Center and Cyclicity Problems: a Computational Algebra Approach, Birkhäuser, Basel, 2009.

[9] B. Sang, B. Huang, Bautin bifurcations of a financial system, Electron. J. Qual. Theory Differ. Equ. 2017(2017), Article ID 95.

[10] Q. Wang, Y. Liu, H. Chen, Hopf bifurcation for a class of three-dimensional nonlinear dynamic systems, Bull. Sci. Math. 134 (2010), 786-798.

[11] Y.A. Kuznetsov, Elements of Applied Bifurcation Theory, Applied Mathematical Sciences, Vol. 112, Springer Verlag, New York, 1998.

[12] E.N. Lorenz, Deterministic nonperiodic flow, J. Atmos. Sci. 20 (1963), 130-141.

[13] R. Eichhorn, S.J. Linz, P. Hänggi, Simple polynomial classes of chaotic jerky dynamics, Chaos Solitons Fractals 13 (2002), 1-15.

[14] J.C. Sprott, Elegant Chaos: Algebraically Simple Chaotic Flows, World Scientific, Singapore, 2010.

[15] A. Algaba, M.C. Domínguez-Moreno, M. Merino, A. J. Rodríguez-Luis, Study of the Hopf bifurcation in the Lorenz, Chen and Lü systems, Nonlinear Dyn. 79 (2015), 885-902.

[16] J. Lü, T. Zhou, S. Zhang, Local bifurcations of the Chen system, Int. J. Bifurc. Chaos 12 (2002), 2257-2270.

[17] C. Ma, X. Wang, Hopf bifurcation and topological horseshoe of a novel finance chaotic system, Commun. Nonlinear Sci. Numer. Simul. 17 (2012), 721-730.

[18] J. Ma, Y. Chen, Study for the bifurcation topological structure and the global complicated character of a kind of nonlinear finance System (I), Appl. Math. Mech. 22 (2001), 1240-1251.

[19] J. Ma, Y. Chen, Study for the bifurcation topological structure and the global complicated character of a kind of nonlinear finance System (II), Appl. Math. Mech. 22 (2001), 1375-1382.

[20] F.S. Dias, L.F. Mello, J.G. Zhang, Nonlinear analysis in a Lorenz-like system, Nonlinear Anal. 11 (2010), $3491-3500$.

[21] M.P. Markakis, P.S. Douris, On the computation of degenerate Hopf bifurcations for $n$-dimensional multiparameter vector fields, Int J. Math. Math. Sci. 2016(2016), Article ID 7658364.

[22] L.F. Mello, M. Messias, D.C. Braga, Bifurcation analysis of a new Lorenz-like chaotic system, Chaos Solitons Fractals 37 (2008), 1244-1255.

[23] L.F. Mello, S.F. Coelho, Degenerate Hopf bifurcations in the Lü system, Physics Lett. A 373 (2009), 1116-1120.

[24] L. Liu, O.O. Aybar, V.G. Romanovski, W. Zhang, Identifying weak foci and centers in the Maxwell-Bloch system, J. Math. Anal. Appl. 430 (2015), 549-571.

[25] D.C. Braga, F.S. Dias, L.F. Mello, On the stability of the equilibria of the Rikitake system, Phys. Lett. A 374 (2010), 4316-4320.

[26] F.S. Dias, L.F. Mello, Hopf bifurcations and small amplitude limit cycles in Rucklidge systems, Electron. J. Differential Equations 2013 (2013), Article ID 48.

[27] J. Llibre, C. Pessoa, The Hopf bifurcation in the Shimizu-Morioka system, Nonlinear Dyn. 79 (2015), 2197-2205.

[28] G. Tigan, On a three-dimensional differential system, Mat. Bilt. 30 (2006), 9-16.

[29] F.S. Dias, L.F. Mello, Analysis of a quadratic system obtained from a scalar third order differential equation, Electron. J. Differential Equations 2010 (2010), Article ID 161.

[30] R. Genesio, A. Tesi, Harmonic balance methods for analysis of chaotic dynamics in nonlinear systems, Automatica 28 (1992), 531-548.

[31] L. Zhou, F. Chen, Hopf bifurcation and Silnikov chaos of Genesio system, Chaos Solitons Fractals 40 (2009), $1413-1422$.

[32] P.T. Cardin, J. Llibre, Transcritical and zero-Hopf bifurcations in the Genesio system, Nonlinear Dyn. 88 (2017), $547-553$.

[33] S.H. Schot, Jerk: the time rate of change of acceleration, Am. J. Phys. 46 (1978), 1090-1094.

[34] O. Umut, S. Yaşar, A simple jerky dynamics, Genesio system, Int. J. Modern Nonlinear Theory Appl. 2 (2013), 60-68.

[35] L.S. Pontryagin, Ordinary Differential Equations, Pergamon Press, London, 1962.

[36] R.S. Irving, Beyond the Quadratic Formula, The Mathematical Association of America, Washington, 2013.

[37] E.J. Barbeau, Polynomials, Problem Books in Mathematics, Springer Verlag, New York, 2003.

[38] E.M. Izhikevich, Equilibrium, Scholarpedia 2 (2007), 2014. 
[39] T. Asada, W. Semmler, Growth and finance: an intertemporal model, J. Macroeconom. 17 (1995), 623-649.

[40] W.M. Liu, Criterion of Hopf bifurcation without using eigenvalues, J. Math. Anal. Appl. 182 (1994), 250-256.

[41] B.D. Hassard, Y.H. Wan, Bifurcation formulae derived from center manifold theory, J. Math. Anal. Appl. 63 (1978), 297-312.

[42] B.D. Hassard, I.M. El-Henawy, Numerical Hopf bifurcation analysis in nonlinear ordinary and partial differential systems for chemical reactor theory, Appl. Math. Comput. 9 (1981), 75-92. 